\title{
LA IDEA DE DIOS EN SCOTTO Y LA METAFÍSICA MODERNA ${ }^{1}$
}

\author{
JUAN A. GARCÍA GONZÁLEZ \\ Universidad de Málaga
}

La teología negativa muestra que es precisamente al referir la inteligencia humana a Dios, o al afrontar cognoscitivamente el tema de Dios, cuando el pensamiento negativo se eleva a sus más altas cotas.

Porque si es difícil conocer a Dios, e incluso en esta vida nunca llegaremos a conocerle entera y adecuadamente, en cambio nos resulta relativamente fácil pensar a Dios; porque más asequible que encontrar a Dios en su propia realidad, como el primer principio de identidad o como la réplica que la persona humana busca, más asequible nos resulta idearlo según esa operación reflexiva y generalizante tan propia de la inteligencia humana.

Y así ocurrió que, cuando a fines de la edad media se redescubrió a Aristóteles, muchos pensadores cristianos de la época encontraron insuficiente su noticia acerca de Dios.

La noesis noeseos aristotélica, en efecto, es el acto puro, motor inmóvil del universo; pero no es creador. Y por tanto no es un ser trascendente, sino sólo el primero de los seres del universo: como el uno neoplatónico del que todo lo múltiple emana. Buscando esa trascendencia, en cambio, el Liber de causis sentenció que prima rerum creatarum est esse, y que Dios está más allá del ser.

Además, el Dios aristotélico es un ser que está encerrado en sí mismo, pues consiste en su propia autognosis; pero carece de voluntad para amar y abrirse a los demás seres, ni siquiera a los espirituales. Fue Agustín de Hipona quien distinguió eros y agapé, para poder atribuir a Dios el amor: un amor de donación; que no sólo explica la providencia divina para con las personas, sino que da razón incluso de la misma creación de todos los seres.

Por estos motivos, muchos pensadores tardomedievales rechazaron lo que Aristóteles sabía de Dios, y como alternativa pensaron cómo tenía que ser el Dios en quien creían: un ser infinito.

\footnotetext{
${ }^{1}$ Este trabajo formaba, junto con otros que han tenido otro destino, una glosa del escrito de Polo Sobre el origen escotista de la sustitución de las nociones trascendentales por las modales (Miscelánea poliana. IEFLP, 9, 2006, 23-31).
} 


\section{a) Dios como ser infinito}

Según Duns Scotto, el hombre tiene un triple conocimiento de Dios ${ }^{2}$ :

- el conocimiento al que llega la razón humana de Dios como el primer principio de todo;

- el conocimiento que reporta la fe cristiana en Dios como el ser omnipotente: no sólo principio del universo que existe; sino capaz de crear otros mundos, de hacer cualquier cosa, y -por tanto- también de intervenir milagrosamente en el curso del universo;

- y el conocimiento que obtendremos en el cielo con la visión de Dios tal y como es en sí mismo: un ser infinito; cuya inacabable contemplación será para el hombre una vida eterna.

De entre ellos, perfectissime conceptus, in quo perfectissime cognoscimus Deum, est conceptus entis infiniti ${ }^{3}$.

Lo que ocurre es que, al apelar a la infinitud divina, se introduce -según Polo- un cierto agnosticismo subrepticio, o un larvado escepticismo ${ }^{4}$. Porque se pierde lo que se sabía acerca de Dios, al estimarlo insuficiente, en lugar de profundizar en ello para mejorarlo. Y se sustituye por una idea negativa de él, que no nos muestra -siquiera incoativamente- su ser real. De la teología negativa, por ello, es fácil pasar a la negación de la teología.

Sin embargo, Scotto piensa en la infinitud de Dios por dos razones: para elevar el ser divino por encima de todo lo creado; y también para localizar en su actividad infinita la unidad de Dios, su esencia propia.

La actividad divina ad extra es la creación; la actividad ad intra es -como la denomina Scotto ${ }^{5}$ la producción de las tres personas divinas. En cambio, Böhme -y el subsiguiente idealismo alemán- asociarán posteriormente ambas dimensiones del actuar divino, incluyendo la creación en un proceso de autogénesis del Dios tripersonal. Pero, con independencia de esta deriva, e incluso de aquella distinción entre ad extra y ad intra, la infinitud -para Scotto- es la característica de la esencia divina considerada en sí misma: essentia tantum; es la unidad del ser divino, al margen de las criaturas exteriores y de la interna distinción de personas.

\footnotetext{
${ }^{2}$ En el prólogo a la Ordinatio III, 206 Scotto distingue la theologia: sive Dei, sive beatorum sive nostra.

${ }^{3}$ DUNS SCOTTO: Ordinatio I, 3, 1.

${ }^{4}$ POLO, L.: Antropología trascendental I: la persona humana. Eunsa, Pamplona 1999; p. 87.

${ }^{5}$ Pluralitas divinarum personarum declaratur ex productione: Ordinatio I, II, 201.
} 
Si de todas las perfecciones del ser divino se puede decir que son infinitas, por aquella dimensión superlativa de la teología de que hemos hablado, entonces la infinitud es el género indeterminado del que las distintas perfecciones son como especies concretas, y es así la unidad de todas ellas. Se aprecia aquí la índole negativa de esta consideración escotista que separa la infinitud como esencia divina.

El antecedente de esta consideración separada de la unidad divina es la doctrina de Avicena ${ }^{6}$ sobre el tercer estado de la esencia. Pues, al modo como esta teoría nos propone considerar la esencia en sí misma, al margen de que esté en el pensamiento o en la realidad, es decir, al margen de su estatuto lógico o físico; así también la infinitud de su actividad constituye la esencia de Dios, al margen de la creación o de la producción de las personas divinas.

Pero entonces, así como cabe sospechar que Avicena está pensando como impensada la esencia cuando la toma en sí misma, así también para Scotto la infinitud divina, y además como un motivo para serlo, está pensada como desbordando la intelección: no ya la humana, pues la capacidad intelectual del hombre es manifiestamente limitada, sino la misma intelección divina; pues ésta remite a la distinción de personas, ya que exige el verbo personal de Dios.

Si el intelecto divino fuera esencial, y no sólo personal, Dios no sería infinito, sino que quedaría definido como un ser espiritual y cognoscente: perfectamente autocognoscente, noesis noeseos, ipsum intelligere subsistens; pero esto -según Scotto- no es la esencia común a las personas divinas, sino sólo la generación de la persona del verbo.

Un planteamiento análogo en este punto al de Scotto (en punto a la separación de la esencia divina respecto de su triple ser personal) encontramos también en el maestro Eckhart. Pues Eckhart recoge de Tomás de Aquino la asimilación de los trascendentales a las personas divinas: el ser al padre, la verdad al hijo y la bondad al espíritu que procede de ambos. Pero la esencia divina es lo unitario del ser divino, porque es común a las tres personas, y ya que Dios es uno sólo. Por eso habla Eckhart de un Deus latens et dormiens, antes de la distinción de las personas. Por lo demás, para Eckhart Dios está también más allá del ser, pues es pura inteligencia: ipsum intelligere subsistens, el mismo entender autosuficiente y no derivado del ser.

${ }^{6}$ Cfr. GONZÁLEZ GINOCCHIO, D.: La metafísica de Avicena: arquitectura de la ontología. Univ. Navarra, Pamplona 2010. 


\section{b) El larvado agnosticismo de Scotto}

Pero insisto en el diagnóstico poliano acerca de la tesis escotista sobre la infinitud divina; y que aquí formulamos así: en lugar de conocer a Dios, Scotto nos propone pensar a Dios. Y pensar en el mejor Dios en que podamos pensar, o del mejor modo que podamos hacerlo: como un ser infinito.

Este cambio se produce para mejorar - presuntamente- la deficiente noticia de Dios que los griegos nos habían transmitido; pero la noción negativa de Dios como ser infinito encubre cierto agnosticismo, y genera sus propios problemas.

Es cierto que Aristóteles no elevó la metafísica hasta su dimensión trascendental; y que con los solos conceptos de la sustancia y su actividad, se llega tan sólo a una noción muy imperfecta de Dios como espíritu motor del cosmos. Pero el descubrimiento de la creación -que Tomás de Aquino formuló señalando el alcance real de la distinción entre la esencia y el ser- permitiría acceder al ámbito trascendental; y entonces entender el ser divino como la plenitud existencial: por la identidad de su ser, que es su propia esencia; y de la que hay que decir que es originaria, improducible en el tiempo. Pero también intelectual: la identidad del Dios cognoscente con su propio conocerse, es decir, con su verbo personal; algo muy distinto y superior a la mera unidad metódico-temática de la noesis noeseos aristotélica. Aquélla es la unidad trascendental, la identidad real; ésta ya la formuló Plotino como una unidad negativa, de indistinción entre el entender y lo entendido.

Cabía profundizar en el conocimiento teológico (de teología filosófica), en lugar de refugiarse en una idea negativa de Dios. Porque, de acuerdo con la distinción entre conocer y pensar, conocer a Dios exige una teología positiva: que acceda al ser divino desde las criaturas; o sea, desde las perfecciones puras del ser de los entes, desde los trascendentales; en lugar de una teología negativa, que, a partir de la idea de infinito, termina por sustituir esas nociones por otras modales, que son las propias del pensamiento generalizante y reflexivo.

\section{c) El argumento ontológico}

Es decir, en lugar de proceder a posteriori: desde las criaturas hacia el creador, con una teología primero negativa, luego positiva y finalmente superlativa; en lugar de eso, Scotto ha procedido a priori, forjando una noción negativa de Dios como ser infinito, desde la cual hablar luego de la creación y de las criaturas. 
Quizás el antecedente de la noción escotista de Dios como ser infinito, especialmente en lo que tiene de un proceder a priori, sea el argumento ontológico ${ }^{7}$ propuesto por Anselmo de Canterbury. Quien precisamente además, parte de la idea de Dios como el máximo ser pensable: la idea que hasta un necio tiene de Dios.

La variación escotista, de la que luego hará un especial uso Leibniz, es reemplazar la idea de máximo pensable por la de máximo posible; Dios no es tanto el ser mayor que el cual no puede pensarse ningún otro, sino más bien el ser mayor que el cual no cabe ningún otro $^{8}$ : porque es el ser infinito. Y -por consiguiente- existe necesariamente, o la existencia conviene necesariamente a su esencia.

A partir de Duns Scotto, la filosofía moderna propende a considerar a Dios como res infinita (Descartes), o como el ser que tiene una infinidad de atributos (Spinoza). Y a conceder legitimidad al argumento ontológico, en sus distintas formulaciones: el ser omniperfecto, que incluye la existencia como una de sus perfecciones; el ser necesario, cuya esencia incluye la existencia; etc.

Me atrevo incluso a sugerir que la consideración escolástica de la aseidad como el constitutivo formal de Dios contiene una derivada de este enfoque apriorístico, o esencialista; porque plantea la cuestión del por qué algo existe -si a se o ab alio- como injustificadamente previa a la misma existencia de los seres, que -sin embargo- es lo realmente primero y principal. Dios existe por sí mismo porque la identidad del ser es originaria y no puede ser producida. Pero eso no significa que su esencia sea existir, o que su esencia incluya la existencia (en ambos casos, prioridad de la esencia); sino más bien que su ser es idéntico con su esencia, o que su existencia es su propia esencia: ipsum suum esse subsistens (con prioridad del ser).

En cambio, en este enfoque apriorístico prima la esencia sobre la existencia; y es una consecuencia de la postura de Avicena que distingue el triple estado de aquélla. Igualmente, la idea de un proceso de autogénesis divina, que sustenta el idealismo alemán, exige cierta antecedencia (per modum finis) de la esencia divina sobre su ser, pues es ella la que con ese proceso se realiza. Y, efectivamente, Hegel ve su propia filosofía como el desarrollo, la formulación extensa, o la demostración, del argumento ontológico.

\footnotetext{
${ }^{7}$ Antes de Kant se lo denominaba argumento a simultáneo; otros lo denominan simplemente, y no sin razón, argumento lógico. Cfr. GONZÁLEZ, A. L.: Teología natural. Eunsa, Pamplona 1985; p. 74.

${ }^{8}$ Con todo, el Proslogion presenta dos distintas formulaciones del argumento. En el capítulo II se habla de Dios como aquello mayor que lo cual nada puede pensarse, mientras que en el capítulo XV se habla de que Dios no sólo es aquello mayor que lo cual nada puede pensarse, sino también algo mayor que lo que puede pensarse. El pensamiento negativo finge autotrascenderse.
} 


\section{d) El problema de la inteligibilidad divina: la idea simbólica de Dios}

El problema de este enfoque escotista acerca de la infinitud como esencia divina es el de la inteligibilidad de Dios; porque, de suyo, la infinitud es una noción negativa, que escapa a la razón. Y no sólo a la humana, como hemos dicho, sino incluso al mismo entendimiento divino: que -por ser posterior y distinto a la infinitud, pues es personal- no la alcanza.

Por ser la noción de infinito una noción negativa, minora el alcance y sentido de nuestro conocimiento de Dios. Con una noción así, más que mostrar -aunque sea incoativamentela esencia divina, parece que todo lo más se lograría una remitencia simbólica de la mente humana a Dios: dirigida hacia él, pero sin llegar a alcanzarlo, sino dejándolo más allá del pensamiento de los hombres.

Descartes planteó así la primera de sus demostraciones de la existencia de Dios. Argumentó, sobre poco más o menos, señalando que la idea de un ser infinito ni es ficticia ni adventicia: ni la hemos forjado nosotros -que somos finitos-, ni la hemos tomado de fuera, pues todo lo que conocemos es finito; sino que es innata, ya que tiene que haber sido puesta en el alma por el propio Dios ${ }^{9}$. Dios, entonces, más que lo conocido con la idea de infinito -cuya realidad no es infinita- es la causa de esa misma idea.

En su libro De Dios que viene a la idea, Levinas ha recogido esta reflexión cartesiana; y señalado, en un parecido orden de consideraciones, el sentido profético de la idea de Dios: tal que anuncia una realidad que desborda su concepto.

Y por su parte Polo entiende también que el conocimiento que el hombre tiene de su propia actividad reflexiva, de esa operación negadora o generalizante que es capaz de ejercer, suscita la idea simbólica de la deidad ${ }^{10}$ : no la indeterminación máxima -que nunca se da-; sino el prius que no puede faltar si hay algo: cualquier objeto ideal, más o menos determinado.

Pero, aun concediendo validez al conocimiento simbólico de Dios, no debería impedir otro conocimiento más adecuado de su existencia. Porque, si la idea de infinito es más bien simbólica y carece de contenido intrínseco, entonces la problemática que lega Scotto al pensamiento moderno es la de la intelección del infinito: porque es exigible una mínima comprensión del ser divino.

\footnotetext{
${ }^{9}$ Cfr. GARCÍA LÓPEZ, J.: El conocimiento de Dios en Descartes. Eunsa, Pamplona 1976; pp. 59 ss.

${ }^{10}$ Cfr. Antropología trascendental, v. II: la esencia de la persona humana. Eunsa, Pamplona 2003; p. 221.
} 


\section{e) Intelecciones modernas del infinito}

Polo sugiere que el voluntarismo y el idealismo modernos constituyen una cierta derivada del planteamiento escotista, y una cierta respuesta al problema planteado por Scotto acerca de la intelección de la infinitud.

Quizás ampliando un poco la sugerencia poliana, me atrevo a sugerir tres posicionamientos modernos acerca de la intelección del infinito:

\section{$\left.1^{\circ}\right)$ El voluntarismo:}

Como la noción de infinito es impensable (o ha de pensarse como tal), el voluntarismo se queda con la idea de que Dios es omnipotente: un descenso al segundo de los grados del conocimiento de Dios distinguidos por Scotto. La omnipotencia es, ciertamente, manifestación de infinitud.

Ser infinito significa, entonces, ser omnipotente; la infinitud no es sustantiva sino adjetiva: lo infinito es el poder de Dios. Pero no del modo como es debido afirmar la omnipotencia divina -compatible con su suprema sabiduría y derivada de su plena y originaria existencia, sino concediendo a Dios un poder primario (Dios es la plenitud del poder, más que del existir $^{11}$ ) y omnímodo: no subordinado a la razón, sino superior a ella; Dios puede hasta lo contradictorio: como hacer un círculo cuadrado ${ }^{12}$, o mandar al hombre que le odie. Es la posición de Ockham, algo más radical que la de Scotto; quien sí parece respetar la vigencia para Dios del principio de contradicción.

La omnipotencia divina así entendida muestra, en su arbitrariedad, su incompatibilidad con la verdad: un desorden entre los trascendentales, como dirá Polo. Y se asimila con la espontaneidad de la voluntad que admite Ockham a partir de la perseitas escotista; y que es una respuesta al determinismo averroísta para abrir un sitio a la libertad humana.

Para el nominalismo, en efecto, la inteligencia del hombre es pasiva, especular; mientras que lo activo en el hombre es la voluntad, que no sigue a la inteligencia sino que se activa por sí misma (per se); de resultas, se revaloriza la acción práctica del hombre. Y de esa manera, es más importante para el hombre amar a Dios que conocerle (máxime cuando el conocimiento no alcanza la infinitud divina); y es más importante la acción que la contemplación.

\footnotetext{
${ }^{11}$ Cfr. CUSA, N.: El Possest. Ed. Angel Luis González. Universidad de Navarra, Pamplona 1998.

${ }^{12}$ De quadratura circuli es una obra escrita por el Cusano en 1450.
} 
Con esta inversión en la consideración clásica de los dinamismos del hombre, tal y como lo enuncia Polo ${ }^{13}$, los nominalistas pretenden abrir un hueco a la subjetividad e interioridad de la persona humana, en la que se ubica la relación con Dios; pero lo consiguen a costa de separarla de la realidad, y de aislarla en sí misma: es el ideal de la autonomía, y de la reforma en el plano religioso.

\section{$\left.2^{\circ}\right)$ El idealismo:}

Para el idealismo, infinito significa todo: Dios es la totalidad del ser.

En Leibniz, la totalidad de la posibilidad, que exige su realización efectiva; y así Dios es causa sui, como dijo Spinoza, o el ser que existe por sí mismo, por su propia posibilidad. Lo enteramente posible, piensa Leibniz, existe necesariamente; es su formulación del argumento ontológico: el conocido como argumento ontológico modal ${ }^{14}$. Y en Hegel Dios es la totalidad de lo pensable; contenido que el concepto necesita para concretarse, de modo que finalmente la idea se conozca a sí misma. Dios es el pensamiento que se piensa a sí mismo, noesis noeseos; pero, concretamente, porque piensa la totalidad de lo pensable.

El idealismo es una cierta réplica al nominalismo, para reponer el valor de la verdad y la dignidad del pensamiento, que puede llegar a Dios y aspirar a lo infinito. Por ello, concede prioridad a la verdad trascendental, que se funda a sí misma independientemente del ser; y que absorbe el bien anulando su mutua distinción. De nuevo, como dice Polo, un desorden entre los trascendentales.

Pero, con todo, el idealismo admite la noción de facticidad nominalista, que se continúa en el empirismo: la reducción de la realidad extramental al individuo singular ininteligible, incoada por la noción escotista de haecceitas ${ }^{15}$. Sólo que postula que el pensamiento negativo reducirá la positividad de lo empírico para lograr el concepto universal, general y total. Lo verdadero es el todo, y el todo es Dios; lo particular, y por serlo, es falso. Loco et numero que definen al individuo singular nominalista, o espacio y tiempo al hecho empírico de la mecánica newtoniana, son la negación de lo lógico, la alienación de la idea; que la dialéctica ha de superar con su aufhebung, es decir, con la negación especulativa.

\footnotetext{
${ }^{13}$ Cfr. POLO, L.: Presente y futuro del hombre. Rialp, Madrid 1993; capítulo III.

${ }^{14}$ Cfr. al respecto GONZALEZ, A. L. (ed.): Las demostraciones de la existencia de Dios según Leibniz. Eunsa, Pamplona 2004²; especialmente el capítulo final.

${ }^{15}$ Término que aparece en sus Reportata parisiensia y en las Quaestiones in libros Metaphysicorum.
} 
Y $3^{\circ}$ ) El nihilismo:

Que está latente en el desorden entre los trascendentales propio de voluntarismo e idealismo, en la medida en que ambos prescinden de la prioridad del ser.

El nihilismo metafísico consiste precisamente en la supresión del primer trascendental: pues la nada nos aparece al negar la entidad. Esta negación late en la noción de infinito; pero, sobre todo, más que en ninguna noción (pues la nada no puede ser estrictamente $\operatorname{algo}^{16}$ pensado), está al alcance de la voluntad: la voluntad se abre a la nada ${ }^{17}$. Por eso el nihilismo es afín al voluntarismo: un rechazo voluntario del ente; y se separa del idealismo, puesto que conlleva también el rechazo de la verdad.

En metafísica, el nihilismo responde al problema de la tematización del infinito diciendo que el infinito no es nada: todo cuanto se da es finito y determinado, luego el infinito es imposible. Ni omnipotencia práctica, ni omniposibilidad teórica: el infinito es lo imposible, lo completamente imposible. Dios no supera la contradicción haciéndola, sino siéndola: siendo contradictorio.

Ello, más que negar la existencia de Dios, pide admitir que para Dios no rige la contradicción. Pero tal cosa se formula sin distinguir el ser divino, como idéntico, del meramente no contradictorio; sino pensando los dos en un mismo plano: es el modo nominalista de ubicar a Dios por encima de la contradicción. Dios existe sin ser nada, o desbordando cualquier determinación.

Este planteamiento constituye una curiosa justificación de lo finito: la noción de fundamento sin fundamento (urgrund que es ungrund), que propuso Böhme. La sucesión de las eventualidades finitas es la manifestación de la imposibilidad de realización del infinito; que no por ello desaparece, sino que impone a las eventualidades su interminable sucesión, su continua mudanza. Como el apeiron de Anaximandro, el logos de Heráclito o el ereignis heideggeriano: pues se trata, en definitiva, del juego de la indeterminación y lo determinado.

Este enfoque, que Polo apunta como presente también en Scotto, y hasta como una manera de salvar su larvado escepticismo, aflora desde luego en el schellingiano escrito sobre la libertad de 1809 (donde Schelling intenta entender la libertad como ese fundamento sin fundamento al que apuntó Böhme). Incluso sugiero que pudiera ser éste el motivo por el que aborta la spätphilosophie, la filosofía positiva del segundo Schelling: pues, en último término, concebir la libertad como abgrund es forjar una noción negativa, y no positiva, de

16 Polo reduce "algo" (el aliquid trascendental) al límite mental: es lo pensado; cfr. Antropología trascendental I, o. c., pp. 61-2.

${ }^{17}$ Aunque así hablamos ya de un nihilismo antropológico, que quizá tiene un sentido especial: cfr. POLO, L.: Antropología trascendental II, o. c., p. 235. 
ella. Y está presente también en el segundo Heidegger, precisamente a partir de su comentario a aquél escrito de Schelling: en su final doctrina sobre la cuadratura ${ }^{18}$. El nihilismo de Nietzsche, que es también voluntarista -y aunque tenga otra muy considerable dimensión antropológica-, también podría inscribirse en esta trayectoria; en particular, si se comprueba -como sugiere Polo ${ }^{19}$ - que hay un frustrado anhelo de infinito en la voluntad de poder, o que la dualidad última del pensamiento nietzscheano no es dionysos-apolo, sino uranos-gea.

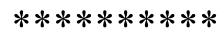

Voluntarismo, empirismo, racionalismo e idealismo, y finalmente nihilismo, son las coordenadas que enmarcan el pensamiento moderno; y que expresan la pérdida del primado del ser propia del realismo antiguo. En su raíz: la discusión nominalista sobre la infinitud divina, y la consiguiente interpretación del obrar humano como espontáneo y de la realidad estramental como ininteligible.

Juan A. García González

jagarciago@uma.es

18 Cfr. ROJAS, A.: La cuadratura. La última palabra del pensamiento ontológico de Heidegger. Universidad, Málaga 2009.

${ }^{19}$ Cfr. Nietzsche como pensador de dualidades. Eunsa, Pamplona 2005; capítulo VIII. 